\title{
What students learn when studying physics practice exam problems
}

\author{
Witat Fakcharoenphol, Eric Potter, and Timothy Stelzer \\ Department of Physics, University of Illinois at Urbana-Champaign, Urbana, Illinois 61801, USA
}

(Received 1 July 2010; published 2 May 2011)

\begin{abstract}
We developed a web-based tool to provide students with access to old exam problems and solutions. By controlling the order in which students saw the problems, as well as their access to solutions, we obtained data about student learning by studying old exam problems. Our data suggest that in general students learn from doing old exam problems, and that having access to the problem solutions increases their learning. However, the data also suggest the depth of learning may be relatively shallow. In addition, the data show that doing old exam problems provides important formative assessment about the student's overall preparedness for the exam and their particular areas of strength and weakness.
\end{abstract}

DOI: 10.1103/PhysRevSTPER.7.010107

PACS numbers: 01.50.H-, 01.40.gb, 01.50.Lc

\section{INTRODUCTION}

In many courses, exams play an important role in student learning as well as assessment. One common method for preparing for an exam is doing "practice" exams. Studying from practice exams has several pedagogical advantages: it provides information about what is "important," it provides formative feedback about students weaknesses, and it can also provide content if information is available such as the correct answer or a solution. Indeed, in a recent survey 93\% of students in the introductory physics course at the University of Illinois said the practice exams were useful to essential in helping them prepare for the exam. Kulik, Kulik, and Bangert [1] showed that students can raise their scores on aptitude and achievement tests by taking practice tests. Balch [2] also showed that doing practice exam helps. The "practice then review solution" group scored higher than the just reviewing the solution group.

Although many of the top students are able to efficiently utilize the opportunities provided by studying old exams, anecdotal evidence suggests weaker students may lack these skills. A common scenario involves a student in a professor's office saying, "The actual exam was nothing like the practice exams. I could do all of the practice problems, but I did terrible on the exam." Further discussion reveals that on the practice tests the student would get a problem wrong, look at the correct answer, and assume that "now I understand the problem." In actuality, the student only knew the final answer to that particular problem-which would most likely not be repeated on the actual exam. So the student's performance on the practice tests was in fact quite consistent with their exam performance. One explanation was observed by Hacker et al. [3]. They showed that high-achieving students

Published by the American Physical Society under the terms of the Creative Commons Attribution 3.0 License. Further distribution of this work must maintain attribution to the author(s) and the published article's title, journal citation, and DOI. were underconfident and low-achieving students were overconfident.

Computer-based practice exams may offer the opportunity to provide more explicit formative assessment for these students. Studies of the efficacy of computer-based systems for the homework stage of learning (e.g., Andes $[4,5]$, Mastering Physics and WebAssign) provide promising results. This study will investigate whether such systems are also beneficial for studying practice exam material. Potential benefits include improved formative assessment and optimized problem selection to improve students' efficiency in preparing for an exam.

By analyzing student performance on old exam problems delivered via the web and subsequent exam performance, this study will provide insights into three important aspects. How much do students learn from doing a multiple-choice exam problem and getting feedback about the correct answer? Does providing students with a complete solution improve their learning? How accurately does student performance on practice exam problems predict their performance on actual exam problems? In particular, can it help predict areas that a student should spend more time studying?

\section{EXPERIMENT DESIGN}

\section{A. Population}

An exam-prep tool was created and made available to students in the introductory calculus based electricity and magnetism course. The calculus based introductory physics courses at the University of Illinois is a "reformed" course emphasizing active learning and utilizing many of the results from physics education research. Students are first introduced to the material through web-based prelectures [6-9] and then asked to answer a few "preflight" questions. These preflight questions and responses are used to inform the lecture as described in just-in-time-teaching [10]. The lecture has a heavy emphasis on peer instruction [11] typically using 5-8 questions in each lecture. In 
addition to the two 50 min lectures each week, students also have a $2 \mathrm{~h}$ discussion section that involves group problems solving of a variety of context rich [12] and tutorial [13] problems. The labs for the course are designed to reinforce the fundamental concepts using the predictobserve-explain format developed by Thornton and Sokoloff [14]. A web-based homework system is used that includes typical exercises as well as interactive examples which provide extensive "Socratic help" [15]. There are three midterm exams in the course which consist of approximately 25 multiple-choice questions, as well as a 50 question comprehensive final exam [16]. The midterm exams are made public immediately after the exam, so exams from prior semesters are available to students as a study aid.

\section{B. Research design}

The exam-prep tool was designed for web delivery and was based on old exam problems. First, exam problems from the past 10 semesters were categorized based on the knowledge needed to successfully complete the problem. The appendix shows a list of the categories used to classify the problems. From this collection, a set of paired problems was selected for each major topic on the exam (Coulomb's law, Gauss' law, electric potential and capacitors) based on the classification of the knowledge. There were a total of 44 questions (22 pairs of problems) roughly evenly distributed among the four topics. The students begin by selecting a general topic they wish to review. They would then be presented with the first of a pair of questions, which we will refer to as iteration 1. After submitting an answer for the first question, they receive feedback on their response, and then go on to the second question, which we will refer to as iteration 2.

In this experiment treatment variations included the order in which the two questions were presented, as well as the nature of the feedback: just the correct answer, or a full solution. Students were randomly assigned to one of four groups. Table I shows the treatments for the four different groups on the first two sets of paired questions.

From the table, each group receives the same net treatment: having access to the full solutions on the first

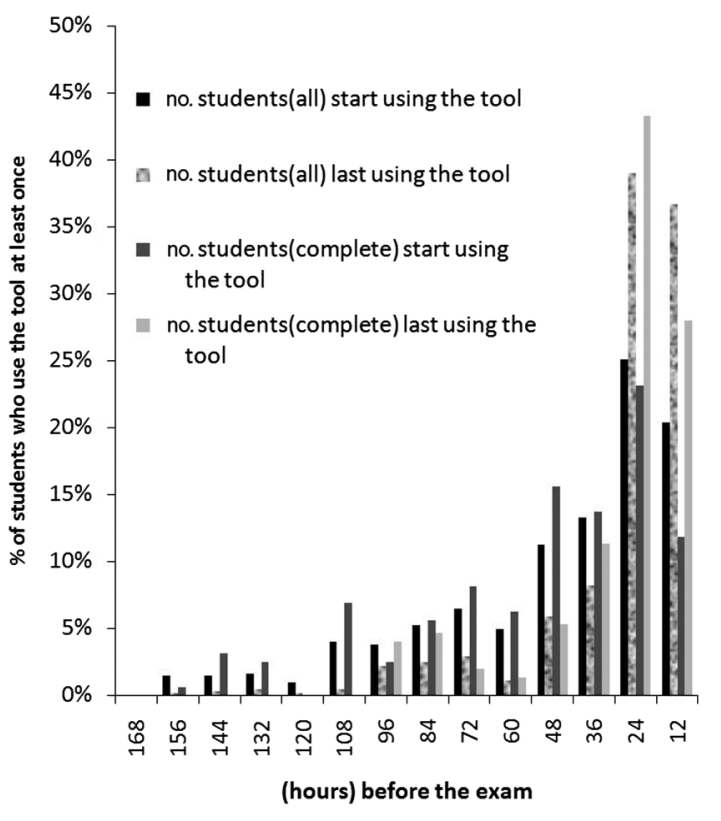

FIG. 1. Student interaction with exam-prep tool. The highest usage was during the $24 \mathrm{~h}$ prior to the exam. Of the 1100 students enrolled in the course, 769 tried at least one question, 494 tried at least half, and 160 did all of the questions.

questions for half of the pairs and on the second questions for the other half of the pairs.

\section{Exam-prep tool}

This tool was made available to students in the Fall 2009 semester for the week before the first hour exam. A link to the tool was provided on the course web site, and an Email was sent to all students inviting them to use the tool and provide feedback.

Figure 1 shows distributions of student interactions with the homework prep tool. Of the 1100 students enrolled in the course, 769 tried at least one question, 494 tried at least half, and $160 \mathrm{did}$ all of the questions. Although some students started using the tool early, the greatest use was within the last $24 \mathrm{~h}$ before the exam.

TABLE I. Order in which problems are seen by four different groups, as well as their access to answers and solutions after answering the question. Four groups of students are required in order to capture students' performance on every problem for all three situations (first of the pair, after its pair with the answer and after its pair with the solution option). Problem A, B, C and D in the table are used to emphasis that all four groups should receive the same amount of answer or solution treatment.

\begin{tabular}{|c|c|c|c|c|c|c|c|c|}
\hline \multirow[b]{3}{*}{ Group } & \multicolumn{4}{|c|}{ Question pair one } & \multicolumn{4}{|c|}{ Question pair two } \\
\hline & \multicolumn{2}{|c|}{ Iteration 1} & \multicolumn{2}{|c|}{ Iteration 2} & \multicolumn{2}{|c|}{ Iteration 1} & \multicolumn{2}{|c|}{ Iteration 2} \\
\hline & Question & Feedback & Question & Feedback & Question & Feedback & Question & Feedback \\
\hline 1 & A & Answer & B & Solution & $\mathrm{C}$ & Solution & $\mathrm{D}$ & Answer \\
\hline 2 & $\mathrm{~A}$ & Solution & B & Answer & $\mathrm{C}$ & Answer & $\mathrm{D}$ & Solution \\
\hline 3 & B & Answer & A & Solution & $\mathrm{D}$ & Solution & $\mathrm{C}$ & Answer \\
\hline 4 & $\mathrm{~B}$ & Solution & A & Answer & $\mathrm{D}$ & Answer & $\mathrm{C}$ & Solution \\
\hline
\end{tabular}


The midterm exam for the course included 13 questions that were selected from old exam problems that matched a pair of problems used on the exam-prep tool.

\section{DATA ANALYSIS}

\section{A. Learning from similar problems}

Apart from any formative assessment, it is interesting to look at what students are able to learn by doing an old exam problem and receiving feedback. In particular, does simply providing the correct answer help students learn? Does providing a full solution significantly improve what they can learn? Data from student interactions with the exam-prep tool provides some insight into these questions.

In order to determine whether students leverage a practice experience (iteration 1) to improve performance on a similar problem (iteration 2), a baseline score for the difficulty of each problem was set using the scores from students seeing the question on iteration 1 . In other words, groups 1 and 2 set the baseline score for questions $\mathrm{A}$ and $\mathrm{C}$, while groups 3 and 4 set the baseline score for questions B and D. One can then compare this baseline result with the performance of the remaining groups on iteration 2, to see the effect of doing a similar problem. The horizontal axis is the baseline score, the vertical axis shows the scores for students seeing the problem in iteration 2. Results are separated based on whether students had access to a brief solution for the problem in iteration 1 or only the answer.

There are several interesting features of this plot. First, it shows that on average students' performance improves from iteration 1 to iteration 2, and having access to the full solution for that problem is in general more beneficial than just having access to the answer. The average score for the groups seeing the problem in iteration 1 was $58.8 \% \pm 0.2 \%$. The average score for students seeing the problem in iteration 2 that only had access to the answer for the problem in iteration 1 was $63.5 \% \pm 0.3 \%$, and the average score for students seeing the problem in iteration 2 that had access to the full solution for the problem in iteration 1 was $66.0 \% \pm 0.3 \%$. Hence, working a similar problem in iteration 1 and having access to the answer resulted in an improvement of $4.7 \% \pm 0.4 \%$, and having access to the full solution resulted in an additional $2.5 \%$ improvement. The uncertainty included in this comparison includes only the variation due to variation in student ability, as the problem pairs were identically matched. Using $\chi^{2}$ analysis, we found that the scores from the group seeing the problem in iteration 1 and the group seeing problem in iteration 2 with access to only the answer are significantly different with $\chi^{2}$ (d.o.f. $\left.=43\right)=80.84$ $(p<0.005)$. Also, the scores from the group seeing the problem in iteration 2 with access to only the answer and with access to the solution are significantly different with $\chi^{2}$ (d.o.f. $\left.=43\right)=59.98(p<0.05)$.

Perhaps the most interesting feature of Fig. 2 is not the net student improvement, but the large variation in student

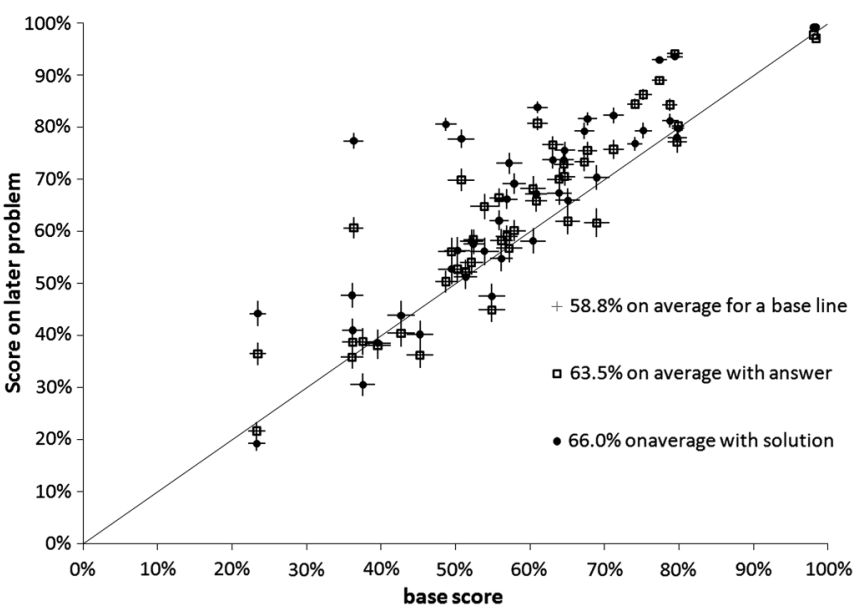

FIG. 2. Results for the 22 groups of paired problems. The horizontal axis is the baseline score for the problem from the two groups that saw the question first, the vertical axis shows performance by students seeing the problem second. These two groups are separated based on if they had access to the complete solution for the paired problem or only the answer. See the appendix for more information.

improvement on the different sets of paired problems. Approximately $3 / 5$ of the problems showed significant improvement (more than $2 \sigma)$ ). Of these, one-third (1/5 of all problems) showed a $10 \%$ or greater improvement (average over both treatments), with 3 problems showing quite dramatic gains of $20 \%-40 \%$. On the other hand, $1 / 3$ of the problems showed no improvement, and 2 of the problems actually had worse performance.

A qualitative analysis of the most improved and least improved problem pairs provides some insight into the opportunities and limitations to what students learn from studying similar problems. From early observation, most of the questions that showed large gains were in problem pairs that have very similar solutions. That is, a relatively straightforward translation of the solution for the first problem could be used to get the answer for the second problem. The appendix shows a typical set of these problems and their corresponding solutions that had a large positive impact. In such cases the answer for the paired problem can be obtained by simply substituting the appropriate charges into the solution for the original problem. It is not surprising that students score significantly higher on the second problem. Upon examining the solutions for all 22 problem pairs, we categorized 5 of the pairs as having virtually identical solutions. The average gain of these pairs is $15 \% \pm 3 \%(18 \% \pm 5 \%$ for the group that could view the solutions and $11 \% \pm 3 \%$ for the group that only had access to the correct answer). The gain on the remaining 17 question pairs is $4 \% \pm 1 \%(5 \% \pm 1 \%$ for the group with access to the solutions and $3 \% \pm 1 \%$ for the group that only had access to the correct answer). Using $\chi^{2}$ analysis, we found that the scores from identical solution problems and the rest are significantly different with 
$\chi^{2}$ (d.o.f. $\left.=1\right)=14.9(p<0.001)$. A useful follow-up question would be to see if the students literally used the result from the original problem, or if they reproduced the answer by following the steps of the solution to the original problem.

What about the questions that did not show a large improvement? A careful examination of these problems suggests two explanations. In some cases the lack of improvement can be attributed to limitations in the question format (e.g., multiple-choice questions that did not have effective distracters, so guessing played an important role). However, some of these responses suggest that the students are getting only a very superficial understanding from the paired problems. The appendix shows the paired problems on Gauss' law pertaining to planar symmetry. The problems look nearly identical at the surface, and were categorized as requiring identical data. However, students actually performed 5\% worse on the paired problem than on the original. It appears students were incorrectly choosing to simply apply the final formula from the problem in iteration 1 to get the answer for the problem in iteration 2. The appendix shows a pair of questions that showed almost no gain. On the surface, the problems look quite similar, a set of capacitors connected to a battery, and the student is asked to solve for the charge or voltage on one of the elements. Furthermore both problems were categorized identically, as they require the same physics knowledge (definition of capacitance and how to combine capacitors in series and parallel). However, the specific values of the components chosen for the problems give them a large amount of symmetry, such that the answer can be surmised without simplifying and expanding the circuit. Indeed, the solution presented used this symmetry to solve the problem. Although both problems can be solved by taking advantage of the symmetry, the method used in the first problem is not directly applicable to the paired problem.

Based on data from the 22 sets of paired problems in this study, we draw the following conclusions. Doing old exam problems can improve a student's performance on subsequent similar problems. Having access to the solutions for the old exam problems can increase that level of improvement. In general this improved performance was restricted to problems with a high degree of similarity, both in the content and solution, suggesting a relatively superficial level of learning. These results may be specific to the format of the questions and the quality of the solutions. However, these results are consistent with our typical instruction strategy. We do not teach courses by simply doing practice exam problems. Instead we begin by providing students with a strong foundation through reading, lecture, recitation, homework, and perhaps labs. Then, once this foundation is set, students refine their understanding in preparation for the exam by studying old exam problems.

\section{B. Formative assessment}

Perhaps the most important value of studying old exam questions is their ability to provide students with formative assessment [17]. The top performing students often report this as a very effective technique. However, lower achieving students may not be as skilled at interpreting their performance on a practice exam. A web-based exam-prep tool may be able to help these students by making the formative assessment more explicit. In particular, the exam-prep tool could use the students' performance on practice problems to assess their overall preparedness for the exam, as well as specific topics that the student would benefit from studying more.

To assess the suitability of old exam problems for providing general and specific formative assessment, we next look at the correlation between student performance on the practice exam questions and on the actual exam. Since the ability to predict student performance will be strongly dependent on the number of questions they do, we restricted this analysis to the 162 students who completed all 44 exam-prep exercises.

Figure 3 shows a scatter plot of the 160 students that completed all 44 exam-prep exercises. On the horizontal axis is their performance on the exam-prep exercises, on the vertical axis is their performance on the exam. There is a statistically significant correlation between the two scores $(r=0.56, p<0.0001)$. This correlation is low compared to the values of $r=0.9$ one would expect if giving two identical exams. However, this is not surprising, considering many students are using this tool at the beginning of their studies (so they can study more and gain improvement outside the tool), and we have already seen

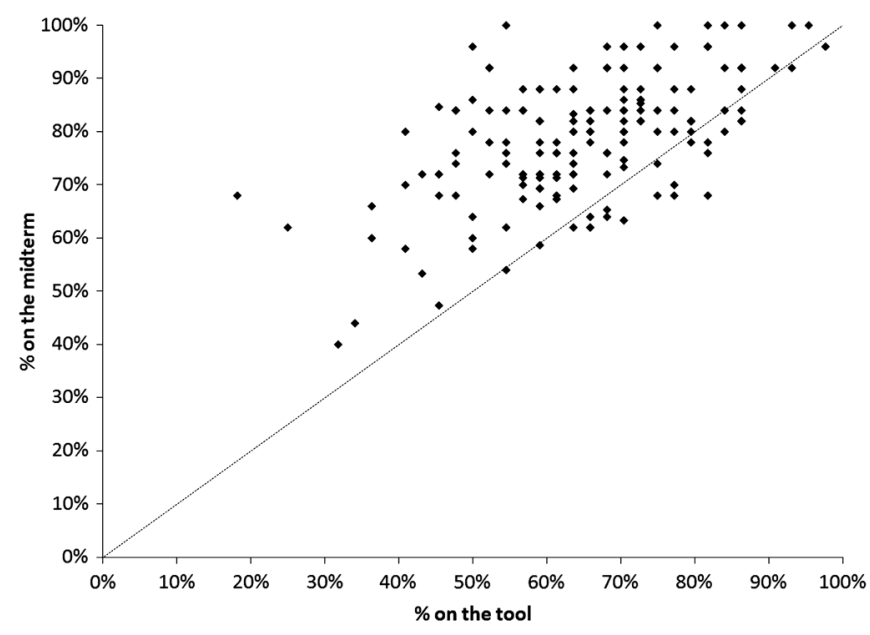

FIG. 3. There are 160 students who answered all problems in the tool. Each diamond represents a student's score on the examprep tool and the exam. The horizontal axis is the students' scores on the practice problems. The vertical axis represents the students' scores on the exam. Most points lie above the dashed line, showing students performed better on the exam than on the exam-prep tool. 


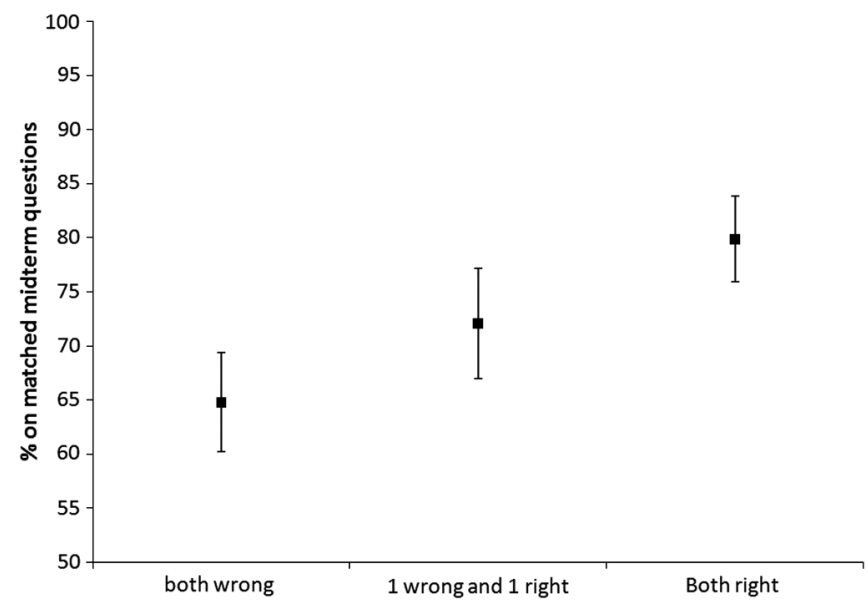

FIG. 4. Thirteen of the questions given on the exam were matched with 13 of the paired problems on the exam-prep tool. Students that got both practice problems correct scored $15 \%$ higher on the matched problem on average than students that got both practice problems wrong.

that studying these practice problems can improve their score. Indeed, it is reassuring to see that although the difficulty of the exam-prep exercises and the exam questions was approximately equal, nearly all students performed significantly higher on the exam than they did on the exam-prep tool. So the tool can be seen to provide a minimum assessment (the dotted line) of the student's preparedness for the exam, with very few students performing significantly below that line on the exam.

In addition to assessing a students general preparedness for the exam, it would be helpful if the tool could identify particular strengths and weaknesses. Such information could be helpful in identifying particular areas that the student should focus on studying. Figure 4 shows a comparison of student performance on exam questions based on how the student performed on the matched practice exam questions. Regardless of the improvement after the use of the tool, students who performed well (answered both problems right) on the tool did about $15 \%$ better on matched midterm problems than students who performed poorly.

One explanation for the above difference is simply that better students do better on both the practice problems and the actual exam. To determine if the exam prep can help differentiate weaknesses in an individual student, we created Fig. 5. The diamonds represent how students perform relative to the rest of the class on topics for which they got both paired problems correct. The boxes show how they performed relative to the rest of the class on topics for which they got both paired problems wrong. The first thing we see is that students using the tool two or more days before the exam are typically stronger, scoring $6 \%$ higher than the average student. We also see there is little predictive power from the tool. This is attributed to the fact these students have time to study their areas of weakness.

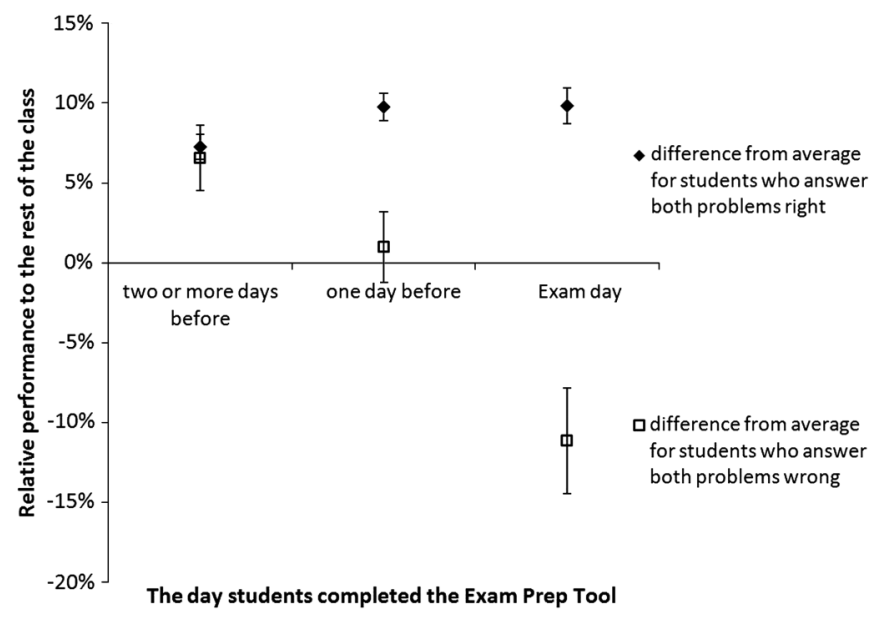

FIG. 5. Student relative performance on exam problems for topics the exam-prep tool predicted they would perform well on (diamonds), compared with topics the tool predicted they would not perform well on (squares). Early on, the predictive power is very weak, perhaps because students had time to work on the material they did not know. However, on the exam day the predictive power was quite good, showing a $20 \%$ difference in student performance.

However, for students using the tool on the day of the exam, there is a dramatic difference in exam performance. They tend to score about $8 \%$ higher than the rest of the class on topics they did well on, and $13 \%$ worse than the rest of the class on topics they did poorly on.

However, this gap also depends on base performance of the students. To minimize any systematic effects (stronger students always perform better) the results are shown in Fig. 6 based on student performance in the mechanics course which on average has a high correlation with performance in the electricity and magnetism course. As shown in the graph below, "A" students performed almost equally, no matter how they did on the tool, consistent with the idea that strong students are able to learn well from practice exams. However the "B" to "D" students show a significant spread based on their performance on the tool. Roughly speaking, "D" students who can answer both problems right will perform as well as " $\mathrm{B}$ " students who answered both problems wrong.

With the same groups of students based on their performance on the mechanic course, we can show the effect of the tool compared to students who did not use the tool. The gain can be observed almost throughout all range of students (except $\mathrm{A}+$ and $\mathrm{D}-$ students). On average, the students who used the tool did about $3.7 \% \pm 1.1 \%$ higher. The point is not to show $3.7 \%$ gain, because it is obviously a small gain for showing students what is on the midterm beforehand. On the previous part, we show that similar problem and full (brief) solution feedback can improve students performance by about $7 \%$ (average over 44 problems) or 5.5\% (over 26 matched pair problems). Hence, this gain of $3.7 \%$ on the midterm is reasonable. 


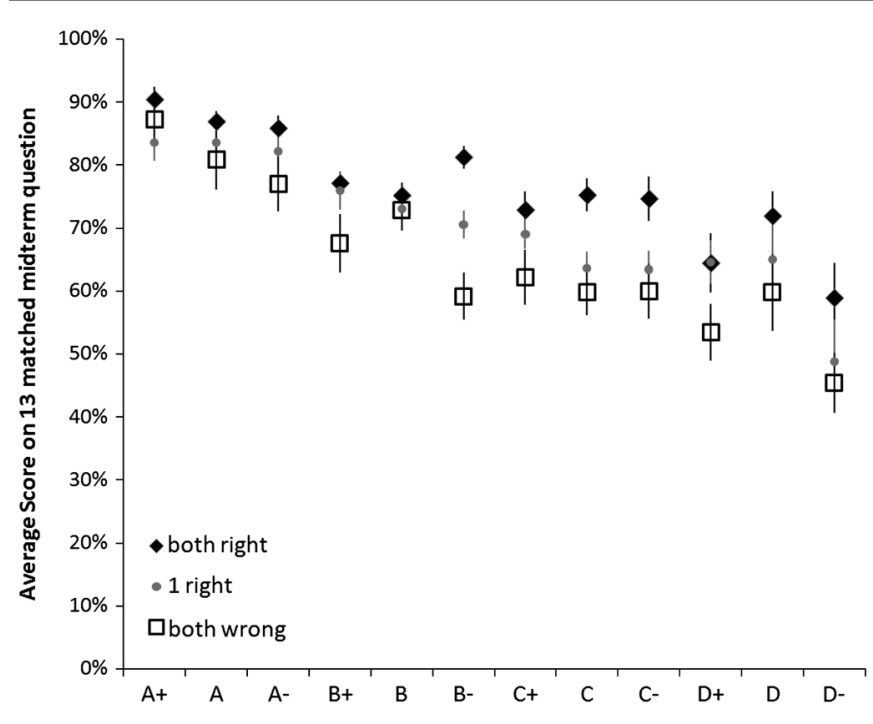

FIG. 6. Student performance on exam problems for topics the exam-prep tool predicted they would perform well on (diamonds), and topics the tool predicted they would not perform well on (squares). The students are binned based on their grade in the prior semester (mechanics) physics course. For B+ through $\mathrm{C}$ - students, performance on the practice tool is a better predictor of performance then their performance in the prior physics course.

\section{DISCUSSION}

Studying old exams is a time-honored technique for preparing for future exams. There are two potential benefits. Perhaps the most important is the formative assessment it provides to the student about what they need to study. In addition it can provide learning opportunity, giving students practice in problem solving, and feedback for problems which they get wrong. In this study, we have created a web-based exam-prep tool that provided some data on how much students learn from studying old exam questions.

The first observation is that students do learn from doing the old exam questions. We saw that on average students performed about $6 \%$ better after doing a similar problem, and having the solution available provided nearly twice the gain relative to having only the answer. However, the wide variation in gain on different paired problems revealed that the level of understanding gained from studying the solutions was relatively superficial. For students with a strong background, this may be all they needed to fill a small gap in their understanding. However, students with a weaker foundation most likely would benefit from a stronger structure of help. It would be interesting to see if providing a deeper level of help, such as a multimedia mini lecture could result in a more consistent learning gain.

The second observation is that student performance on the exam-prep tool can provide important formative assessment, both on overall preparedness as well as on specific areas of weakness. In general, students that did well on the practice problems also did well on the exam. Since students may have further studied after the practice exams, the correlation was relatively modest $(r=0.56)$. This is consistent with the fact that students' performance on the exam was almost always better than their performance on the practice problems, even though the problems were of comparable difficulty. Furthermore, the practice exam problems accurately reflected the students relative strength in the various topics. Students that did well on a particular topic on the practice exam typically scored $20 \%$ higher than students that did poorly on that topic on the practice exam.

There have been studies on practice exams with two instructional hopes: better self-awareness of how prepared the students are and better performance on the real exam. Bol and Hacker [18] showed that high-achieving students with nonidentical practice exam treatment can evaluate their performance more accurately than those with no treatment. However, their students (both high- and lowachieving students) with a practice exam did not perform better on the real exam, whereas better performance on a real exam was reported from using both computer-based assessment [19,20] and paper-based assessment [21].

The self-selection effect suggested by Dunn and Mulvenon [22] can cause misinterpretation on results from some practice exam experiments. If the practice exam is presented as optional, it is possible that highachieving students were more likely to participate in the optional exercise than low-achieving students. This can void the results from many experiments if participants and nonparticipants are compared without any initial constrains. Unlike other experiments on practice exams, our experiment was set to avoid the self-selected effect. All of our participants were randomly assigned to one of the four groups with equal treatment. Each participant's answers contributed to all three condition types (prior problems, later problems with solution of the prior problem, and later problems with just the answer of prior problem). The score comparison between the three condition types should not be affected by the self-selected effect. Also, in formative assessment analysis, all the midterm scores that we analyzed were only from participants who completed those matched pair problems. However, it is possible to compare between participants and nonparticipants and avoid the self-selected effect. By comparing scores of students within the same level of performance (same mechanic course grades), the gain of $3.7 \%$ from using the tool should be minimally affected by self-selection.

There are limitations of using multiple-choice practice exams, but the interpretation of our results should not be affected due to our experiment setting. The score from multiple-choice problems can be affected by the choices in the problem as the context of the problem itself. The score can be saturated from random guessing or getting rid of choices. Negative effect can also be observed, students 
can be trapped by common mistake choices (in our experiment). However, Scott, Stelzer, and Gladding showed that students' scores from carefully constructed multiplechoice problems can be reliable and consistent with written exam scores [16]. Also, in our experiment, we compared the scores from the same problems that appeared in different order (prior or later). All the multiple-choice effects should be canceled out with normal subtraction between later and prior scores (at least to the first order). However, the validity of the gain size from the subtraction is variable. We cannot say that $(50 \%-70 \%)$ gain from a 5-choiced problem is better or worse than $(30 \%-45 \%)$ gain from a 4-choiced problem.

In future studies, we will investigate if it is possible to use this information to help students more efficiently learn the material. The first improvement would be to simply point out their areas of weakness, so they could focus their area of study. More advanced features of an exam-prep tool could include providing different forms of content, from highly structured solutions to mini lectures. The ultimate goal of this guidance will be not just to help the students learn the material, but to help them understand the process of using the formative assessment to guide their learning.

\section{ACKNOWLEDGMENTS}

We would like to thank Adam Feil for creating the examprep tool computer system.

\section{APPENDIX: LIST OF CATEGORIES AND PROBLEM SETS}

See separate auxiliary material for a list of categories as well as a typical problem set and solutions.
[1] J. Kulik, C. Kulik, and R. Bangert, Effects of practice on aptitude and achievement test scores, Am. Educ. Res. J. 21, 435 (1984).

[2] W. Balch, Practice versus review exams and final exam performance, Teach. Psychol. 25, 181 (1998).

[3] D. Hacker, L. Bol, D. Horgan, and E. Rakow, Test prediction and performance in a classroom context, J. Educ. Psychol. 92, 160 (2000).

[4] A. Gertner and K. VanLehn, Andes: A coached problem solving environment for physics, Lect. Notes Comput. Sci. 1839, 133 (2000).

[5] K. VanLehn, K. Lynch, K. Schulze, J. A. Shapiro, R. Shelby, L. Taylor, D. Treacy, A. Weinstein, and M. Wintersgill, The Andes Physics Tutoring System: Lessons learned, Int. J. Artif. Intell. Educ. 15, 147 (2005).

[6] T. Stelzer, G. Gladding, J. Mestre, and D. T. Brookes, Comparing the efficacy of multimedia modules with traditional textbooks for learning introductory physics content, Am. J. Phys. 77, 184 (2009).

[7] T. Stelzer, D. T. Brookes, G. Gladding, and J. P. Mestre, Impact of multimedia learning modules on an introductory course on electricity and magnetism, Am. J. Phys. 78, 755 (2010).

[8] Z. Chen, T. Stelzer, and G. Gladding, Using multimedia modules to better prepare students for introductory physics lecture, Phys. Rev. ST Phys. Educ. Res. 6, 010108 (2010).

[9] To experience these prelectures as would a student, please visit https://online-s.physics.uiuc.edu/courses/phys212/ gtm/No_Login/page.html.

[10] G. Novak, E. Patterson, A. Gavrin, and W. Christian, Justin-Time Teaching: Blending Active Learning with Web Technology (Prentice-Hall, Upper Saddle River, NJ, 1999).
[11] E. Mazur, Peer Instruction: A User's Manual (PrenticeHall, Upper Saddle River, NJ, 1997).

[12] P. Heller and M. Hollabaugh, Teaching problem solving through cooperative grouping. Part 2: Designing problems and structuring groups, Am. J. Phys. 60, 637 (1992).

[13] L. McDermott and P. Shaffer, Tutorials in Introductory Physics (Prentice-Hall, Upper Saddle River, NJ, 2002).

[14] R. Thornton and D. Sokoloff, Assessing student learning of Newton's laws: The force and motion conceptual evaluation and the evaluation of active learning laboratory and lecture curricula, Am. J. Phys. 66, 338 (1998).

[15] To experience the interactive examples, please visit http:// research.physics.illinois.edu/per/ie.html.

[16] M. Scott, T. Stelzer, and G. Gladding, Evaluating multiple-choice exams in large introductory physics courses, Phys. Rev. ST Phys. Educ. Res. 2, 020102 (2006).

[17] P. Black and D. Wiliam, Assessment and classroom learning, Assess. Educ. Princ. Pol. Pract. 5, 7 (1998).

[18] L. Bol and D. Hacker, A comparison of the effects of practice tests and traditional review on performance and calibration, J. Exp. Educ. 69, 133 (2001).

[19] J. Gretes and M. Green, Improving undergraduate learning with computer-assisted assessment, J. Res. Tech. Educ. 33, 46 (2000).

[20] G. Johnson, College student use and relationship to achievement, Can. J. Learn. Tech. 32 (2006).

[21] K. Knaus, K. Murphy, and T. Holme, Designing chemistry practice exams for enhanced benefits: An instrument for comparing performance and mental effort measures, J. Res. Tech. Educ. 86, 827 (2009).

[22] K. Dunn and S. Mulvenon, A critical review of research on formative assessment: The limited scientific evidence of the impact of formative assessment in education, Practical Assess. Res. Eval. 14, 8 (2009). 